\title{
Motor stereotypies in adult patients with Tourette syndrome
}

\author{
Maneeka Ubhi ${ }^{1}$, Kanu Achinivu ${ }^{1}$, Stefano Seri ${ }^{2}$ \& Andrea E Cavanna*, 1,2,3 \\ ${ }^{1}$ Michael Trimble Neuropsychiatry Research Group, University of Birmingham \& BSMHFT, Birmingham, UK \\ ${ }^{2}$ School of Life \& Health Sciences, Aston University, Birmingham, UK \\ ${ }^{3}$ Sobell Department of Motor Neuroscience \& Movement Disorders, Institute of Neurology \& University College London, UK \\ *Author for correspondence: a.e.cavanna@bham.ac.uk
}

\begin{abstract}
Aim: Correctly diagnosing repetitive behaviors in patients with Tourette syndrome (TS) can be challenging. The differential diagnosis between tics and stereotypies is of particular importance, because of treatment implications. Methods: We assessed the prevalence and clinical characteristics of stereotypies in a large sample of adult patients with TS attending a specialist clinic. Results: Mild stereotypies were reported by $21 / 148$ patients (14.2\%). Patients with stereotypies were significantly more likely to have a comorbid diagnosis of Asperger syndrome, attention-deficit and hyperactivity disorder, and obsessive-compulsive disorder, compared with patients without stereotypies. Multiple linear regression analysis revealed that the presence of Asperger syndrome significantly predicted stereotypy severity. Conclusion: Stereotypies are not rare in adults with TS and other neurodevelopmental conditions, especially Asperger syndrome.
\end{abstract}

First draft submitted: 25 December 2019; Accepted for publication: 5 February 2020; Published online: 11 May 2020

Keywords: asperger syndrome • autism spectrum disorder • stereotypies • tics • Tourette syndrome

Tourette syndrome (TS) is a neuropsychiatric disorder characterized by the chronic presence of multiple motor and vocal tics since childhood [1,2]. Tics are defined as sudden, rapid, recurrent, nonrhythmic movements and vocalizations that tend to wax and wane in both frequency and intensity over time [3,4]. Tics are characteristically preceded by subjective urges, in other words, distressing sensations which are temporarily relieved by tic expression (e.g., eye blinking, shoulder shrugging, throat clearing) [5]. The diagnosis of TS is based on clinical presentation, history and direct observations of tics [6]. TS is frequently associated with other conditions; it has been consistently shown that about $90 \%$ of patients with TS fulfill the diagnostic criteria for comorbid psychiatric disorders, most commonly obsessive-compulsive disorder (OCD) and attention-deficit and hyperactivity disorder (ADHD) [7,8].

Patients with TS can present with different types of repetitive behaviors in addition to tics [9,10]. Previous research has mainly focused on the differential diagnosis between tics and obsessive-compulsive symptoms [7]. More recently, the challenges involved in the differential diagnosis between tics and functional movement disorders have been highlighted [11]. Stereotypies (e.g., head nodding, arm flapping, body rocking) are different types of repetitive behaviors, defined as involuntary, coordinated, patterned, repetitive, rhythmic, purposeless but seemingly purposeful or ritualistic movements, postures or utterances' [12]. Stereotyped repetitive behaviors such as finger tapping are present in the healthy population and repetitive movements are considered normal in developing children [13,14]. In addition to primary stereotypic movement disorder, stereotypies are frequently reported in the context of autism spectrum disorders (ASD), mental retardation and sensory deprivation [15-17], however, little is known about their prevalence and clinical correlations in TS [18]. Stereotypies have a more rhythmic nature than tics and, importantly, are not characterized by suppressibility or associated with subjective urges. Moreover, stereotypies can have an earlier age of onset than tics (as early as 3 years vs 5-7 years), although in clinical practice there is a considerable degree of overlap. Distinguishing between tics and stereotypies based solely on observation can be difficult, especially in consideration of the dynamic tic repertoire of patients with TS across the lifespan $[6,16,19,20]$. Identifying the specific type of repetitive behavior in TS is crucial to reach a correct diagnosis and to provide patients with accurate information about their prognosis, as well as to implement appropriate treatment strategies [4]. Unlike tics, stereotypies do not tend to improve with age, show poor response to pharmacotherapy,

Future Medicine 
and are therefore managed with specific behavioral approaches [21]. It is important to note, however, that tics show significant variability in response to treatment interventions. We set out to conduct the first study on the prevalence and clinical characteristics of stereotypies in adult patients with TS.

\section{Methods}

\section{Patients}

The participants were consecutive adult patients attending a specialist outpatient clinic for TS at the Department of Neuropsychiatry (Birmingham and Solihull Mental Health NHS Foundation Trust and University of Birmingham, UK) between 2010 and 2015. All participants had a diagnosis of TS according to the current DSM criteria [1]. Patients with an uncertain diagnosis and with severe learning disability were excluded from the study. A total of 148 patients were included in the study. The average age of the participants was 30.0 years (SD 12.3). Upon recruitment, all participants provided written informed consent and the research project was granted ethics approval from the NHS ethics committee and the Research and Innovation Department at BSMHFT (2010).

\section{Psychometric measures}

Both demographic and clinical data, including age of tic onset, family history of tics, medication, number of tics, presence of psychiatric comorbidities (namely obsessive-compulsive symptoms and full-blown OCD, ADHD, ASD, learning disability, affective disorders and anxiety disorders) were collected using the National Hospital Interview Schedule for TS [22]. Stereotypies in the context of TS were prospectively diagnosed by the same movement disorders expert based on clinical assessment and history taking, focusing on the recommendations set out by the European Society for the Study of TS: age at onset, temporal pattern, movement type, rhythm, duration, premovement sensory-motor phenomena, trigger, suppressibility and family history [6]. In clinical practice, the degree of overlap in age at onset and treatment response invalidates the use of these variables for the differential diagnosis between tics and stereotypies. Moreover, analyzing a particular movement among many others can be difficult at times. We have therefore made use of observations beyond the examination period, including home video recordings produced by the patients and their carers. A clinical description of the type(s) of stereotypy was also collected from each patient. Two clinician-rated instruments were used to measure the severity of tics and stereotypies: the Yale Global Tic Severity Scale (YGTSS) and the Stereotypy Severity Scale (SSS). It is important to note that the SSS, like the YGTSS on which it is based, is not useful for diagnosing the movement in question, but for rating its severity if appropriate. Moreover, the SSS does not have the amount of validation data that have been produced for the YGTSS.

The YGTSS is a validated measure of tic severity for use in adults and children with tic disorders [23,24]. Tic severity is rated according to five parameters: number, frequency, intensity, complexity and interference (each scored $0-5)$. Motor and vocal tics are rated separately (both scored 0-25). Overall impairment, encompassing difficulties in self-esteem, family life, social acceptance and school or job functioning, is scored 0-50. The maximum total YGTSS score is 100, with higher scores indicating higher tic severity.

The SSS is a measure of stereotypy severity developed based on the YGTSS $[13,25]$. The rated categories include number (scored $0-3)$, frequency $(0-5)$, intensity $(0-5)$ and interference $(0-5)$, with a total score of 18 . Overall impairment, encompassing self-esteem, family school or social acceptance, is scored 0-50. The maximum total SSS score is 68 , with higher scores indicating higher stereotypy severity.

\section{Statistical analysis}

Patients with and without stereotypies were compared with regard to demographic and clinical data, including tic severity. For continuous variables, an independent t-test or its nonparametric equivalent (Mann-Whitney $\mathrm{U}$ test), was performed to assess differences between the groups. The $\chi^{2}$ test (Fisher's exact test for small groups) was used to assess intergroup differences in categorical variables. The relationship between YGTSS and SSS scores was explored using Spearman's rho correlation, as the SSS scores were not normally distributed. A multiple linear regression analysis was performed to identify which factors predicted stereotypy severity (SSS scores). All clinical and demographic variables were entered into the regression model. p-values below 0.05 were considered significant. Data analyses were carried out using SPSS version 20.0 for Windows. 
Table 1. Comparison of demographic and clinical characteristics in adults with Tourette syndrome, with and without stereotypies.

\begin{tabular}{|c|c|c|c|}
\hline Characteristic & $\begin{array}{l}\text { Tourette syndrome without stereotypies } \\
(n=127)\end{array}$ & Tourette syndrome with stereotypies $(n=21)$ & p-value \\
\hline Mean age (years) & 30.6 & 26.1 & 0.180 \\
\hline Male $(n, \%)$ & $86(67.7 \%)$ & $16(76.2 \%)$ & 0.437 \\
\hline Mean age at tic onset (years) & 7.6 & 7.3 & 0.735 \\
\hline Family history of tics (n, \%) & $63(49.6 \%)$ & $12(57.1 \%)$ & 0.522 \\
\hline OCS $(n, \%)$ & $57(44.9 \%)$ & $13(61.9 \%)$ & 0.148 \\
\hline$O C D(n, \%)$ & $35(27.6 \%)$ & $11(52.4 \%)$ & $0.023^{\dagger}$ \\
\hline $\operatorname{ADHD}(n, \%)$ & $19(15.0 \%)$ & $16(76.2 \%)$ & $0.001^{\dagger}$ \\
\hline Asperger syndrome (n, \%) & $0(0.0 \%)$ & $4(19.0 \%)$ & $0.001^{\dagger}$ \\
\hline Mild learning disability (n, \%) & $7(5.5 \%)$ & $1(4.8 \%)$ & 1.000 \\
\hline Affective disorders (n, \%) & $41(32.3 \%)$ & $4(19.0 \%)$ & 0.222 \\
\hline Anxiety disorders (n, \%) & $17(13.4 \%)$ & $1(41.8 \%)$ & 0.471 \\
\hline Tic medication ( $\mathrm{n}, \%)$ & $83(65.4 \%)$ & $11(52.4 \%)$ & 0.253 \\
\hline Mean number of tics & 20.6 & 29.6 & 0.153 \\
\hline YGTSS total motor score (mean) & 15.91 & 15.33 & 0.457 \\
\hline YGTSS total vocal score (mean) & 11.39 & 12.43 & 0.388 \\
\hline YGTSS total tic score (mean) & 27.30 & 27.76 & 0.857 \\
\hline YGTSS total score (mean) & 52.26 & 54.95 & 0.491 \\
\hline
\end{tabular}

\section{Results}

The majority of participants were male $(102 / 148,68.9 \%)$ and the mean YGTSS score was 52.6 (SD 16.5), indicating moderate-to-marked tic severity. Comorbid OCD was present in 46/148 patients (31.1\%), ADHD in 35/148 (23.7\%) and Asperger syndrome in 4/148 (2.7\%). The prevalence of stereotypies in our sample was $21 / 148(14.2 \%)$ and $76.2 \%$ of patients with stereotypies were males. The mean SSS score in the 21 patients found to have stereotypies was 19.7 (SD 8.5). Seventeen patients had one stereotypy, three had two stereotypies and one patient had three different stereotypies. Most stereotypies involved the head and upper limbs. The types of reported stereotypies were as follows: bilateral hand shaking $(n=8)$, body rocking $(n=4)$, head nodding $(n=4)$, bilateral arm waiving $(n=4)$, bilateral hand flapping $(n=4)$, head shaking $(n=2)$.

There were no significant differences between the groups of patients with and without stereotypies in terms of demographic characteristics. With regard to clinical characteristics, we found that patients with TS and stereotypies were more likely to have comorbid Asperger syndrome ( $\mathrm{p}=0.001)$, ADHD ( $\mathrm{p}=0.001)$, and OCD ( $\mathrm{p}=0.023)$ (Table 1).

There was no significant association between SSS and YGTSS scores (or YGTSS subscores), indicating no relationship between stereotypy and tic severity $(r=-0.019, \mathrm{p}=0.933)$. Multiple linear regression analysis revealed the presence of Asperger syndrome as a significant predictor of SSS scores $\left(F(1,19)=29.933, R^{2}=0.612, p=\right.$ $0.001)$.

\section{Discussion}

We conducted the first systematic assessment of stereotypies in a large sample of adult patients with TS. Our findings confirmed that motor stereotypies are frequently encountered in specialist TS clinics across the lifespan. In our sample, $14.2 \%$ patients reported stereotypies in association with their tic symptoms. Most of the existing studies on stereotypies have been conducted in child populations and data on adult patients without severe learning disability are lacking [13]. Previous research on healthy children with stereotypies [21] found a male predominance (63\%), which is similar to our findings (76.2\%). Previous data on stereotypy severity are limited, however research on nonautistic children with stereotypies found mean SSS scores of 40.5 (before behavioral therapy) and 29.6 (after-treatment intervention) [25]. Both scores are higher than stereotypy severity scores reported by our sample, suggesting that stereotypies in adults with TS tend to be relatively mild. Moreover, the lack of correlation between 
SSS and YGTSS ratings provides further evidence in support of the clinical distinction between stereotypies and tics. The most commonly reported stereotypies in our sample involved the face and upper limbs. The clinical characteristics of stereotypies were in line with previous findings in child populations: in a study of 40 children diagnosed with stereotypies, the most frequent combinations of stereotypies were hand flapping and hand shaking, clenching hands and facial grimacing, and rocking while grimacing [21]. When prompted to focus on the subjective experiences accompanying their repetitive movements, most patients were able to clearly differentiate between tics and stereotypies, based on the presence of the characteristic premonitory urge to tic.

We found that adults with both tics and stereotypies were more likely to have comorbid Asperger syndrome, $\mathrm{ADHD}$, and OCD compared with adults with tics without stereotypies. We also found that the presence of Asperger syndrome was a strong predictor of stereotypy severity. This could reflect the presence of stereotypies (alongside preoccupations, rigidity and restricted interests) among the key criteria for the diagnosis of ASD [1,26]. Moreover, the high prevalence of stereotypies in patients with ASD has been demonstrated in several studies, including a large study on 277 children recruited from clinics and special schools with autism and nonautistic developmental disorders. The prevalence of stereotypies in this clinical population was $44 \%$, confirming that stereotypies are common symptoms of ASD [27]. As well as being the most frequent comorbidities in TS, both ADHD and OCD have been reported in association with stereotypies [28]. The results of previous clinical studies have shown that $50 \%$ of children with stereotypies can present with an additional neuropsychiatric diagnosis [29] and the prevalence of comorbid ADHD and OCD can be as high as 25 and 5\%, respectively [21].

Our study has limitations. First, all participants were recruited from a specialist center, where patients with higher tic severity and multiple psychiatric comorbidities can be over-represented (referral bias). Second, the relatively small number of patients with stereotypies identified in our study may not be representative of the wider community of patients with TS. Third, the large discrepancy in sample size between patients with and without stereotypies could have affected the results of the statistical analyses. Finally, patients with severe learning disability, including autism, were excluded from the study, possibly resulting in underestimation of the prevalence of stereotypies in TS.

\section{Conclusion}

In conclusion, mild stereotypies are not rare in adults with TS and are more likely to occur in patients with psychiatric comorbidities, particularly Asperger syndrome, who require careful assessment for the differential diagnosis of their repetitive behaviors. Our findings have practical implications, as a better understanding of the clinical presentation of stereotypies in patients with TS can help preventing the misdiagnosis of these symptoms as refractory tics, as well as the implementation of inappropriate treatment interventions. Further studies on the prevalence and clinical characteristics of stereotypies in larger samples of patients with TS, both in specialist clinics and in the community, are needed to replicate our findings. Comparing adult and children populations and examining whether stereotypies can affect health-related quality of life in TS would be important areas of future research.

\section{Future perspective}

Correctly diagnosing repetitive behaviors in patients with TS can be challenging, as a number of patients present with different motor manifestations alongside their tics. The differential diagnosis between tics and stereotypies is of particular importance for future research, because of the treatment implications. Mild stereotypies are not rare in adults with TS and are more likely to occur in patients presenting with other neurodevelopmental conditions, especially Asperger syndrome, who require careful assessment for the differential diagnosis of repetitive behaviors.

\section{Acknowledgments}

The authors are grateful to R Holder for statistical advice. Gratitude is also expressed to Tourettes Action-UK and Tourette Association of America for their continuing support.

Financial \& competing interests disclosure

The authors have no relevant affiliations or financial involvement with any organization or entity with a financial interest in or financial conflict with the subject matter or materials discussed in the manuscript. This includes employment, consultancies, honoraria, stock ownership or options, expert testimony, grants or patents received or pending, or royalties.

No writing assistance was utilized in the production of this manuscript. 


\section{Ethical conduct of research}

Upon recruitment, all participants provided written informed consent and the research project was granted ethics approval from the NHS ethics committee and the Research and Innovation Department at BSMHFT (2010). The authors state that they have obtained appropriate institutional review board approval or have followed the principles outlined in the Declaration of Helsinki for all human or animal experimental investigations. In addition, for investigations involving human subjects, informed consent has been obtained from the participants involved.

\section{Open access}

This work is licensed under the Attribution-NonCommercial-NoDerivatives 4.0 Unported License. To view a copy of this license, visit http://creativecommons.org/licenses/by-nc-nd/4.0/

\section{Summary points}

- The differential diagnosis between tics and stereotypies is of particular importance, because of the treatment implications.

- This study assessed the prevalence and clinical characteristics of stereotypies using validated psychometric measures in a large sample of adult patients with Tourette syndrome attending a specialist clinic.

- Specific stereotypies, mainly involving the head and upper limbs, were reported by 21/148 patients (14.2\%) and tended to be mild in severity.

- Patients with stereotypies were significantly more likely to have a comorbid diagnosis of Asperger syndrome $(p=0.001)$, attention-deficit and hyperactivity disorder $(p=0.001)$ and obsessive-compulsive disorder $(p=0.023)$, compared with patients without stereotypies.

- Multiple linear regression analysis revealed that the presence of Asperger syndrome significantly predicted stereotypy severity $(p=0.001)$.

- Mild stereotypies are not rare in adults with Tourette syndrome.

- Stereotypies are more likely to occur in patients presenting with other neurodevelopmental conditions, especially Asperger syndrome.

\section{References}

Papers of special note have been highlighted as: $\bullet$ of interest

1. American Psychiatric Association. Diagnostic and Statistical Manual of Mental Disorders (5th Edition). American Psychiatric Association Press, Washington, DC, USA (2013).

2. Cavanna AE, Termine C. Tourette syndrome. Adv. Exp. Med. Biol. 724, 375-383 (2012).

3. Hassan N, Cavanna AE. The prognosis of Tourette syndrome: implications for clinical practice. Funct. Neurol. 27, 23-27 (2012).

4. Cohen SC, Leckman JF, Bloch MH. Clinical assessment of Tourette syndrome and tic disorders. Neurosci. Biobehav. Rev. 37, 997-1007 (2013).

5. Eddy CM, Cavanna AE. Premonitory urges in adults with complicated and uncomplicated Tourette syndrome. Behav. Modif. 38, 264-275 (2014).

6. Cath DC, Hedderly T, Ludolph AG, Stern JS, Murphy T, Hartmann A. European clinical guidelines for Tourette syndrome and other tic disorders. Part I: assessment. Eur. Child Adolesc. Psychiatry 20, 155-171 (2011).

7. Cavanna AE. Gilles de la Tourette syndrome as a paradigmatic neuropsychiatric disorder. CNS Spectr. 23, 213-218 (2018).

- Reviews the multifaceted behavioral spectrum of Tourette syndrome.

8. Cavanna AE. The neuropsychiatry of Gilles de la Tourette syndrome: the état de l'art. Rev. Neurol. 174, 621-627 (2018).

9. Kompoliti K, Koetz CG. Hyperkinetic movement disorders misdiagnosed as tics in Gilles de la Tourette syndrome. Mov. Disord. 13, 477-480 (1998).

10. Ridley RM. The psychology of perseverative and stereotyped behaviour. Prog. Neurobiol. 44, 221-231 (1994).

11. Ganos C, Martino D, Espay AJ, Lang AE, Bhatia KP, Edwards MJ. Tics and functional tic-like movements: can we tell them apart? Neurology 93, 750-758 (2019).

12. Edwards MJ, Lang AE, Bhatia KP. Stereotypies: a critical appraisal and suggestion of a clinically useful definition. Mov. Disord. 27, 179-185 (2012).

- Reviews the definition and clinical characteristics of stereotypies.

13. Singer H. Motor stereotypies. Semin. Pediatr. Neurol. 16, 77-81 (2009).

14. Sanger TD, Chen D, Fehlings DL et al. Definition and classification of hyperkinetic movements in childhood. Mov. Disord. 25, 1538-1549 (2010). 
15. Bodfish JW, Symons FJ, Parker DE, Lewis MH. Varieties of repetitive behaviour in autism: comparisons to mental retardation. J. Autism Dev. Disord. 30, 237-243 (2000).

16. Bonnet C, Roubertie A, Doummar D, Bahi-Buisson N, Cochen de Cock V, Roze E. Developmental and benign movement disorders in childhood. Mov. Disord. 25, 1317-1334 (2010).

17. Kurlan R. A clinically useful definition of stereotypies. Mov. Disord. 28, 404 (2013).

18. Tan A, Salgado M, Fahn S. The characterization and outcome of stereotypic movements in nonautistic children. Mov. Disord. 12, 47-52 (1997).

19. Cavanna AE, Robertson MM, Critchley HD. Catatonic signs in Gilles de la Tourette syndrome. Cogn. Behav. Neurol. 21, 34-37 (2008).

20. Singer HS. Motor control, habits, complex motor stereotypies, and Tourette syndrome. Ann. NY Acad. Sci. 1304, 22-31 (2013).

- Reviews the most commonly reported repetitive behaviors in patients with Tourette syndrome.

21. Mahone EM, Bridges D, Prahme C, Singer HS. Repetitive arm and hand movements (complex motor stereotypies) in children. J. Pediatrics 145, 391-395 (2004).

22. Robertson MM, Eapen V. The National Hospital Interview Schedule for the assessment of Gilles de la Tourette syndrome. Int. J. Meth. Psychiatry Res. 6, 203-226 (1996).

23. Leckman JF, Riddle MA, Hardin MT et al. The Yale Global Tic Severity Scale: initial testing of a clinician-rated scale of tic severity. J. Am. Acad. Child Adolesc. Psychiatry 28, 566-573 (1989).

- Presents the development and validation of the most widely used tic severity assessment tool.

24. Storch EA, Murphy TK, Fernandez M et al. Factor-analytic study of the Yale Global Tic Severity Scale. Psychiatry Res. 149, 231-237 (2007).

25. Miller JM, Singer HS, Bridges DD, Waranch HR. Behavioural therapy for treatment of stereotypic movements in nonautistic children. J. Child Neurol. 21, 119-125 (2006).

26. Freeman RD, Soltanifar A, Baer S. Stereotypic movement disorder: easily missed. Dev. Med. Child Neurol. 52, $733-738$ (2010).

27. Canitano R, Vivanti G. Tics and Tourette syndrome in autism spectrum disorders. Autism 11, 19-28 (2007).

28. Barry S, Baird G, Lascelles K, Bunton P, Hedderly T. Neurodevelopmental movement disorders: an update on childhood motor stereotypies. Dev. Med. Child Neurol. 53, 979-985 (2011).

29. Harris KM, Mahone EM, Singer HS. Nonautistic motor stereotypies: clinical features and longitudinal follow-up. Pediatr. Neurol. 38 , 267-272 (2008). 